\title{
High-cycle fatigue device for low stiffness components
}

\author{
Olivier ARNOULD ${ }^{1}$ and François HILD
}

\author{
LMT-Cachan
}

ENS Cachan / CNRS-UMR 8535 / Université Paris 6

61 avenue du Président Wilson, F-94235 Cachan Cedex, France

${ }^{1}$ Corresponding author. Now at LMGC/Université Montpellier 2 (France). Fax: +33 467144792.

Email: olivier.arnould@lmgc.univ-montp2.fr

\begin{abstract}
Long-term reliability of a high precision pressure sensor using very low stiffness bellows (e.g., some N/mm) made of electroplated $\mathrm{Ni} / \mathrm{Cu} / \mathrm{Ni}$ layered material (overall thickness of the order of $50 \mu \mathrm{m}$ ) is sought. The bellows are expected to stay in service for many decades. Their high cycle fatigue behavior has to be known. A specific setup, which is put in a resonant machine that is displacement-controlled, has been designed. It allows for the simultaneous analysis of eleven samples at a frequency of about $100 \mathrm{~Hz}$. The characterization of the high cycle fatigue behavior of any very thin samples in the 1-10 million cycles range is made possible. In the case of the bellows, fatigue criteria such as airtightness loss are monitored accurately together with a possible stiffness change with the number of cycles.
\end{abstract}

\section{Introduction}

The analysis of very thin samples (i.e., with a thickness less than $0.1 \mathrm{~mm}$ ) subjected to cyclic loadings is not an easy task since conventional testing machines are meant to analyze millimeter to decimeter specimens. In particular, the fatigue study of electroplated nickel is performed by using LIGA (German acronym for LIthographie, Galvanoformung und Abformung) made samples [Madou, 2002]. Either tensile tests with positive load ratios [Cho et al., 2003; Allameh et al., 2004; Aktaa et al., 2005] or flexural tests with fully reversing load conditions [Mohr and Strohrmann, 1992; Boyce et al., 2004; Lou et al., 2005] are performed on (sub)millimeter samples. In the present case, the long-term reliability of small electroplated bellows made of a thin $\mathrm{Ni} / \mathrm{Cu} / \mathrm{Ni}$ layered material (overall thickness $\approx 50 \mu \mathrm{m}$, Fig. 1) is sought [Arnould and Hild, 2002]. The design criteria are related to their stiffness and airtightness as they are used to convert a (very) small pressure variation into a displacement in a high precision pressure sensor. Yet, in the above-mentioned cases, the airtightness cannot be assessed easily. It is proposed to develop a special setup to directly test bellows in their “environment” (i.e., they 
are welded onto A304L(N) stainless steel holders (Fig. 1a)). Furthermore, as high cycle fatigue behavior is sought, i.e., at least 1 to 10 million cycles are necessary to obtain the endurance properties, up to ten samples may be tested simultaneously to save time.

Section 2 is devoted to the principle of the setup put in a resonant testing machine. The design of the main part of the setup is discussed in Section 3 together with its dynamic behavior. In Section 4, the validation procedure is presented. Last, initial results from the bellows are discussed in Section 5.

\section{Setup principle}

The airtightness of the bellows welded on stainless steel holders is the main fatigue criterion for this application. Consequently, it is easiest to test the component itself. At least ten samples must be tested for each loading step (i.e., applied displacement amplitude $u_{a}$ ) to obtain the required data, namely, the displacement-fatigue life plot for airtightness loss. This curve may be used to identify a probabilistic fatigue model [Arnould et al., 2004; 2005]. The main features of the bellows are its very low stiffness (i.e., $2.65 \pm 0.21 \mathrm{~N} / \mathrm{mm}$ ), its maximum inservice displacement amplitude $u_{a}$ of $0.5 \mathrm{~mm}$ (with a zero mean value $\bar{u}=0$ ) and its fundamental (axial) frequency estimated by finite element computations (i.e., axisymmetric geometric model with shell finite elements) to be of the order of $5 \mathrm{kHz}$.

A reconditioned resonant electromagnetic fatigue machine (Amsler Vibrophore 2 HFP 421, Fig. 2a) is chosen to perform the experiment. Its working frequencies vary from 35 to $240 \mathrm{~Hz}$ with a maximum load range of $10 \mathrm{kN}$ and an uncertainty less than 1\%. The maximum displacement amplitude is $0.6 \mathrm{~mm}$ [Zwick, 2006]. This machine functions on the basis of the resonance principle using the natural frequency of the tested sample associated with a mass. The basic mechanical system (Fig. 2b) consists of a variable mass $m_{v}$ for frequency tuning, a sample of negligible mass and stiffness $k_{s}$, a load cell of negligible mass and stiffness $k_{l}$ and a flexible joint of stiffness $k_{j}$. Resonance is obtained and maintained thanks to a magnet with an angular frequency $\omega=\sqrt{\left(k_{j}+k^{\prime}\right) / m_{v}}$ with $1 / k^{\prime}=1 / k_{s}+1 / k_{l}$. The mean load level is obtained by the deformation of the flexible joint fixed between the mobile part of the magnet and the Vibrophore frame. Its deformation is obtained and controlled by the motion of the crosshead. A feedback provides closed loop control of the load amplitude and mean value over the full working range of the Vibrophore.

A specific setup is designed to fit in the Vibrophore instead of the usual sample (maximum available dimensions: length $\times$ diameter $\approx 360 \mathrm{~mm} \times 300 \mathrm{~mm}$, Fig. 2c). Its principle consists in testing $\ell$ bellows of mean 
stiffness $k_{b}$ in parallel with a well-characterized guiding spring of stiffness $k_{g}$ significantly greater than $\ell \times k_{b}$. The dynamic response of the system is thus mainly controlled by the stiffest component, i.e., the guiding spring. It allows one to prescribe bellows displacements, even though the experiment itself is load-controlled, and to be insensitive to the number of bellows or their stiffness changes. The guiding spring links the mobile part of mass $m_{m}$ (in dark grey, Fig. 2c) of the setup to its frame of mass $m_{f}$ (in light grey, Fig. 2c). The frame is fixed on the load cell of the Vibrophore whereas the mobile part is linked to the magnet.

The built setup is shown in Figs. 3 and 4a. Its design is inspired by the mechanical parts of shakers. The guiding spring is made of two sets of stacking disks fixed together by an inner and outer clamp. In each clamp, disks are separated by annular spacers and the stack is held by a sufficient number of screws to ensure a uniform applied pressure between disks and spacers. To avoid fretting [Giannakopoulos et al., 1998], a thin polymeric film of molybdenum disulfide (Molykote D321R coating and G Rapid Plus paste from Dow Corning) is applied to spacers. Moreover, the disk shape has been chosen in order to obtain a high ratio between the guiding spring radial stiffness and its axial one. It allows one to obtain a pure tensile loading of the bellows without any transverse shear or torsion. Furthermore, the axisymmetric configuration of the setup ensures that all bellows are equally loaded. Four columns link all the fixed parts of the setup, namely, the frame, the outer clamps of the guiding spring and an upper plate to maintain one end of the bellows. The mobile part is made of an inner shaft, inner clamps of the guiding spring and the lower plate onto which the other end of the bellows is fixed. A free core LVDT (050HR016 with ATA2001 conditioning unit from Schaevitz, linear range of $\pm 1.27 \mathrm{~mm}$, calibrated with $1 \mathrm{~mm}$ and $0.5 \mathrm{~mm}$ shims), to avoid wear by friction and to have a high frequency response (i.e., frequency response: $-3 \mathrm{~dB}$ down at $500 \mathrm{~Hz}$ ), is mounted in one of the available sample locations. It measures the relative displacement between the lower and upper plates (Fig. 4b). During the fatigue test, the usual load controlled loop of the Vibrophore is performed. An outer loop is added to check the measured displacement amplitude. The load amplitude is corrected step by step by an integral stage on the error of the displacement amplitude signal. No lock-in is performed for the displacement mean value as it is obtained by the static displacement of the crosshead that is assumed to remain fixed during the test. It is worth noting that, as this system is statically indeterminate, clearances have been added and jigs have been used to align its pieces during its assembly.

Figure 5a shows the detail of a simple fixture of the bellows on the setup. The bellows are welded onto two holders (Fig. 6) that are linked to the upper and lower plates. Since the airtightness is one of the investigated parameters, a pressure sensor (DP2-41E from Sunx with a time response better than $2.5 \mathrm{~ms}$ and a range of 0$100 \mathrm{kPa}$ ) is used and connected in parallel to all bellows by a pneumatic fitting. A light overpressure is applied 
prior to the experiment that stops as soon as a pressure drop is observed. The true stiffness of one bellows and its change with the number of cycles is evaluated in situ thanks to a piezoelectric load cell (9203 low level force sensor with 5015A conditioning unit from Kistler) and a specific fixture (Fig. 5b). The load cell-conditioning unit resolution is of the order of $0.05 \mathrm{~N}$ for $\pm 10 \mathrm{~N}$ of range. The load cell rigidity is of the order of $40 \mathrm{~N} / \mu \mathrm{m}$ and its natural frequency is of the order of $27 \mathrm{kHz}$.

\section{Setup Design}

First, the disks of the guiding spring are designed. The geometry and the material of the disk could be both optimized to obtain the best properties [Ashby, 2005]. However, to simplify their forming and to reduce cost, they are cut in a spring sheet metal (i.e., hot rolled high carbon steel, ASTM 1080). The essential elements to determine their dimensions are the maximum available diameter (of the order of $300 \mathrm{~mm}$ ) to fit in the Vibrophore, the overall stiffness to be as large as possible (compared to that of the bellows) and compatible with the maximum load range $(10 \mathrm{kN})$, and the maximum displacement range for the bellows $(0.5 \mathrm{~mm})$, i.e., $\ell \times k_{b}<<k_{g}<2 \cdot 10^{4} \mathrm{~N} / \mathrm{mm}$. Their first eigen mode should have a frequency greater than the maximum working one whereas the first eigen mode of the whole setup must fit within the working frequency range of the Vibrophore (35-240 Hz). The number of disks, $n$, must be large enough to allow one to tune the setup frequency with a given minimum step (i.e., tuning using the variable mass $m_{v}$ is not possible in the present case).

Each disk is modeled as an axisymmetric shell of inner radius $R_{i}$, outer radius $R_{o}$ (Fig. 3a) and thickness $e$, made of an isotropic elastic material with Young's modulus $E$, Poisson's ratio $v$ and mass density $\rho$. They are assumed to be perfectly clamped, i.e., without any slip. Considering only one disk, an axial load $F$ applied to the inner clamp leads to a displacement $u$ that is estimated by using a small perturbation analysis of shells, i.e., plane stress assumptions and first order deformation [Timoshenko and Woinowsky-Krieger, 1959]. This assumption is satisfied as the maximum displacement of the disk is less than $1 \mathrm{~mm}$ whereas its radial dimension is of the order of some $100 \mathrm{~mm}$. The stiffness $k=F / u$ of one disk thus reads

$$
k=\frac{\pi E e^{3}}{12\left(1-v^{2}\right) R_{o}^{2}}\left[\frac{r^{2}-1}{16 r^{2}}-\frac{\ln ^{2} r}{4\left(r^{2}-1\right)}\right]^{-1},
$$

with $r=R_{o} / R_{i}>1$ and $k_{g}=n \cdot k$. A maximum principal stress criterion is used to check endurance. It is equal to the radial stress on the surface and at the internal radius of the disk 


$$
\sigma_{r r}=\frac{E e}{8\left(1-v^{2}\right) R_{o}^{2}} \frac{2 r^{2} \ln r}{r^{2}-1}\left[\frac{r^{2}-1}{16 r^{2}}-\frac{\ln ^{2} r}{4\left(r^{2}-1\right)}\right]^{-1} u,
$$

that has to be less than 200 MPa for the chosen material. The analytical expression of the first natural (axial) frequency, $\omega_{d}$, of one disk cannot be obtained easily. However, a simplified dimensional analysis [Barenblatt, 1987] leads to

$$
\omega_{d}=\sqrt{\frac{E}{\rho}} \frac{e}{R_{o}^{2}} \psi(r)
$$

with $\psi(r) \approx[0.15 \ln (r)-0.25]^{-1}$ a function determined, in the present case, by finite element calculations (i.e., axisymmetric geometric model with shell finite elements) for $1.5 \leq r \leq 2.5$.

The outer radius $R_{o}$ is finally taken equal to $130 \mathrm{~mm}$ (i.e., the maximum possible value when taking the width of the clamp into account) so that a larger number of bellows may be tested simultaneously (i.e., twelve locations are available at most but one of them is used for the free core LVDT, Fig. 4b). Equation (2) shows that the thinner the sheet, the smaller the stress for a given displacement $u$. By increasing the number of disks, $n$, for a given overall stiffness $k_{g}$, it allows one to decrease the individual sheet thickness ( $k$ is an increasing function of $e^{3}$, see Eq. (1)) and to increase the fatigue lifetime. Moreover, it allows one to improve the axisymmetric response of the guiding spring, as the elastic behavior of hot rolled sheet metal is generally orthotropic, by randomly distributing the disks in-plane orientation in each stack. However, a thickness decrease induces a reduction of the resonance frequency as $\omega_{d}$ is an increasing function of $e$ as well (Eq. (3)). Furthermore, numerical computations of Eqs. (1) to (3) show that the stiffness $k$, the radial stress $\sigma_{r r}$ and the first natural angular frequency $\omega_{d}$ are all decreasing functions of $r$ (thus increasing with $R_{i}$ ). A compromise between stiffness, stress level, resonance frequency and sheet thickness availability is obtained by using $2 \times 5$ disks, each with a thickness of $1.5 \mathrm{~mm}$ and an inner radius $R_{i}=69 \mathrm{~mm}$. The corresponding stiffness is $k=2 \mathrm{kN} / \mathrm{mm}$ per disk (i.e., $\ell \times k_{b} \approx 10^{-3} k_{g}$ ), with a maximum principal stress of 170 MPa for a displacement of $0.5 \mathrm{~mm}$ obtained with an overall load of $10 \mathrm{kN}$. The first resonance frequency of each individual disk is slightly greater than $1 \mathrm{kHz}$ (i.e., more than four times the maximum working frequency).

The dynamic response of the whole system is then analyzed using a simplified approach in which all parts of the setup, except the guiding spring, are assumed to be rigid and damping in the guiding spring, mainly due to disk / spacer friction and air flow through the vents (Fig. 3b), are not accounted for. Note that the diameter and length of the vents have been coarsely chosen to have a Helmholtz resonance frequency [Rayleigh, 1916] 
greater than the working frequency. The Vibrophore frame is assumed to be motionless (its average total weight is greater than $2 \mathrm{t}$ ) and rigid. The stiffness of the bellows is neglected in comparison with all the others. The displacements are assumed to be vertical. The system (Figs. 2b and c) is described as a force-driven harmonic oscillator of two masses $m_{f}$ and $m_{m}+m_{v}$ with two springs, $k_{l}$ and $k_{j}$ respectively, (strongly) coupled by the spring $k_{g}$ [Meirovitch, 2001]. The axial positions of the setup frame and mobile part are measured with respect to their static equilibrium position. They are denoted by $u_{f}$ for mass $m_{f}$ and $u_{m}$ for mass $m_{m}+m_{v}$ (Fig. 2c). The mass $m_{m}+m_{v}$ is loaded by the force $F(t)=F_{a} \cos (\omega t)$ of the magnet. Newton's second law applied to each moving mass reads

$$
\left[\begin{array}{cc}
m_{f} & 0 \\
0 & m_{v}+m_{m}
\end{array}\right]\{\ddot{U}\}+\left[\begin{array}{cc}
k_{l}+k_{g} & -k_{g} \\
-k_{g} & k_{j}+k_{g}
\end{array}\right]\{U\}=\left\{\begin{array}{c}
0 \\
F
\end{array}\right\},
$$

with $\{U\}^{t}=\left\{\begin{array}{ll}u_{f} & u_{m}\end{array}\right\}$. The natural angular frequencies are the two positive roots of the following equation

$$
\left(k_{l}+k_{g}-m_{f} \omega^{2}\right)\left[k_{j}+k_{g}-\left(m_{v}+m_{m}\right) \omega^{2}\right]-k_{g}^{2}=0
$$

They are expressed as

$$
\omega_{ \pm}^{2}=\frac{1}{2}\left[\omega_{1}^{2}+\omega_{2}^{2}+k_{g} / m_{e q}\right] \pm \frac{1}{2} \sqrt{\left[\omega_{1}^{2}+\omega_{2}^{2}+k_{g} / m_{e q}\right]^{2}-4\left[\omega_{1}^{2} \omega_{2}^{2}+k_{g}\left(\frac{\omega_{1}^{2}}{m_{m}+m_{v}}+\frac{\omega_{2}^{2}}{m_{f}}\right)\right]},
$$

with $\omega_{1}^{2}=k_{l} / m_{f}, \omega_{2}^{2}=k_{j} /\left(m_{m}+m_{v}\right)$ and $m_{e q}=m_{f} \cdot\left(m_{m}+m_{v}\right) /\left(m_{f}+m_{m}+m_{v}\right)$. The lowest and first frequency $f_{-}=\omega_{-} / 2 \pi$ corresponds to $u_{m}=\lambda \cdot u_{f}$ with $\lambda$ a positive constant (i.e., the so-called acoustic mode of the coupled harmonic oscillator)

$$
\lambda=\frac{k_{l}+k_{g}-m_{f} \omega_{-}^{2}}{k_{g}} .
$$

This mode is prescribed by the Vibrophore (i.e., loading frequency equal to the first resonance frequency $f_{-}$) and controlled in displacement $\left(u_{m}-u_{f}\right)$ through the free core LVDT measurement. We assume that $\lambda$ is close to the value obtained in the force-driven case as it will be shown hereafter that damping is small (see Section 4). Let us note that $u_{f}$ must be negligible, as much as possible, with respect to $u_{m}$ to reduce the displacement of the setup frame. The second angular frequency $\omega_{+}$corresponds to $u_{m}=\lambda^{\prime} \cdot u_{f}$ with $\lambda^{\prime}$ a negative constant (i.e., the so-called optical mode of the coupled harmonic oscillator) that is calculated by replacing $\omega_{-}$by $\omega_{+}$in 
Eq. (7). This second mode and any displacement of the setup frame induce artifacts that need to be corrected (see Section 4). The effect of this mode is minimized by ensuring that $f_{+}=\omega_{+} / 2 \pi$ is greater than the working frequency $f_{-}$, i.e., $f_{+} / f_{-}$must be as high as possible. The only parameters that can be modified easily are $m_{f}$ and $k_{g}$, as the other stiffness is a property of the Vibrophore, and $m_{m}+m_{v}$ is mainly limited by the possible value for $m_{v}$. A numerical study shows that $f_{+} / f_{-}$reaches a minimum for a given value of $m_{f}$ and $k_{g}$, and that $\lambda$ is a decreasing function of $m_{f}$ and $k_{g}$. Then, $m_{f}$ and $k_{g}$ have to be minimized. However some constrains exist, that is, $m_{f}$ cannot be too small as a sufficient rigidity of the frame must be preserved and $k_{g}$ is mainly limited by the bellows stiffness. Finally, $k_{g}=13.7 \mathrm{kN} / \mathrm{mm}$ (measured value for eight disks, see Section 4) and $m_{f}=33 \mathrm{~kg}$. Other various stiffnesses are determined with quasi-static loadings, namely, $k_{l}=84 \mathrm{kN} / \mathrm{mm}$ and $k_{j}=2.6 \mathrm{kN} / \mathrm{mm}$. The masses are such that $m_{v}=32.5 \mathrm{~kg}$ (its minimum value) and $m_{m}=10 \mathrm{~kg}$. With these values, Eqns. (6) and (7) give $f_{-}=91.7 \mathrm{~Hz}, f_{+} / f_{-}=3, \lambda=6.3$ and $\lambda^{\prime}=-0.12$. Let us note that the best way to reduce artifacts, i.e., to minimize $u_{f}$, may be to rely on replacing the Vibrophore load cell by a stiffer one (e.g., piezoelectric load cell) and by improving the material choice, in particular for the setup frame, in order to minimize its mass by keeping, or improving, its stiffness and its fatigue behavior [Ashby, 2005].

\section{Validation and correction}

The mechanical response was first assessed when the system is loaded statically in a conventional servohydraulic testing machine. The relative displacement of the two plates is measured by two quasi-static LVDTs (Fig. 7a). The applied load is measured by using the load cell of the testing machine (range: $\pm 50 \mathrm{kN}$ ). The response of the system is shown in Fig. 7b. A small non-linearity is observed (i.e., a linear fit of the curve is obtained with a regression coefficient better than 0.99 ) and induced by structural effects associated with microslip in the clamp. A third degree polynomial describes very accurately the experimental data and a total stiffness, i.e., the initial slope, of $17.1 \mathrm{kN} / \mathrm{mm}$ is obtained for ten disks. This value is in good agreement with the a priori linear estimate in small perturbation analysis (i.e., $20 \mathrm{kN} / \mathrm{mm}$ with Eq. (1)) in which perfect clamping conditions were assumed. This validates the simplified approach used in Section 3 to determine the disks stiffness. A slight hysteresis due to damping is also observed and is mainly induced by microslip between disks and spacers. This induces a slight 
heating of the clamp, and of the setup (i.e., about $1^{\circ} \mathrm{C}$ at one sample location, see Section 5), which is observed by thermal measurements [Arnould et al, 2005].

The setup is then installed in the Vibrophore. Only eight disks were finally used to avoid a first natural frequency (Eq. (6) yields $98.8 \mathrm{~Hz}$ when $k_{g}=17.1 \mathrm{kN} / \mathrm{mm}$ ) close to a multiple of the power frequency (50 Hz). The fundamental frequency, $f_{-}$, is then equal to $93 \mathrm{~Hz}$ and $f_{+} / f_{-}$is equal to 2.97 (measured by Fast Fourier Transform of the time-displacement curve of the free core LVDT). This is in good agreement with the theoretical values obtained at the end of Section 3 and validates the simplified approach used for the dynamic behavior of the setup. In particular, it also indicates that damping is small as already shown by analyzing the quasi-static response of the setup.

The following check consists in measuring the vibration modes of the system by visualizing the relative displacements of two markers (Figs. 8a and b) with a strobe light and a long distance microscope. These markers are mounted at one of the sample locations. No rotational or transverse relative displacement modes are observed. This is confirmed by installing accelerometers on different parts of the setup. These results validate the fact that a pure tensile load is applied to the bellows. A final validation, before any experiment on bellows, consists in analyzing the setup behavior when samples with equivalent stiffness as the bellows are installed. Ten mock-up samples are used to validate the experimental procedures (Fig. 8a). The bellows are replaced by a spring with a higher stiffness $(5 \mathrm{~N} / \mathrm{mm})$. As expected, the resonance frequency of the setup is not altered. Finally, by checking the displacements measured by the free core LVDT in five different sample locations during $8 \mathrm{~h}$, the maximum variation of the peak-to-peak displacement between these five positions is equal to $10 \mu \mathrm{m}$ (corresponding standard deviation: $3 \mu \mathrm{m}$ ). It is deemed satisfactory.

The fact that the setup frame is not fixed and that there exists a second eigen mode requires special care when the sample load signal is analyzed. When the piezoelectric load cell is used, the bellows are not directly linked with the upper plate (Figs. 5b and 8c). The measured force $F_{m}$ is made up of two terms, that is, the force $F_{b}$ due to the bellows, or mock-up, elongation and an inertia force

$$
F_{m}=F_{b}+m_{e} \ddot{u}_{f},
$$

where $m_{e}$ denotes the effective mass of the mobile parts attached to the load cell, i.e., the upper holder, the pneumatic fitting for the bellows (Figs. 5b) and an unknown part of the load cell. A corrected load measurement equal to the raw measurement minus $m_{e} \ddot{u}_{f}$ is computed on-line, with a numerical oscilloscope, by measuring $\ddot{u}_{f}$ with an accelerometer installed close to the load cell (Fig. 8c). Unfortunately, $m_{e}$ is not easy to evaluate $a$ 
priori. However, the frequency spectrum of the raw loading measurement determined with the oscilloscope (Fig. 9a) shows a non-negligible component of the load signal at the second natural frequency of the setup. This is mainly due to $\ddot{u}_{f}$ (i.e., $\lambda^{\prime}=-0.12$ so $u_{f}$ is about ten times greater than $u_{m}$ at the second natural frequency, see Section 3). Then, the mass $m_{e}$ is tuned such that the amplitude of the second eigen mode, in the frequency spectrum of the corrected measurement, is minimized (i.e., as small as possible). A value of $37.5 \times 10^{-3} \mathrm{~kg}$ is obtained with the mock-up samples. The fact that it is close to the "static" mass of the upper holder $\left(32 \times 10^{-}\right.$ ${ }^{3} \mathrm{~kg}$ ) is an indirect validation of the identification procedure. The effective mass may contain parts of the load cell whose total weight is about $13 \times 10^{-3} \mathrm{~kg}$. If the correction is used, a good agreement between the static and dynamic measurements is obtained (Fig. 9b). This last correction is implemented in an in-house software so that the output is the corrected load level. This last step validates the whole experimental procedure.

\section{Cyclic experiments on bellows}

To mount the bellows in the setup, special care should be exercised. Handling the bellows may damage them prior to the experiment. Furthermore, their initial length is not identical. Each bellows ends are welded onto calibrated holders that are positioned with a specific mounting shaft (Fig. 6). Each shaft length is machined precisely (better than one hundredth of a millimeter) according to the actual height of the bellows and holders dimensions. The bellows are inserted and fixed onto the two plates of the fatigue setup with these shafts so that they are almost stress free. There initial elongation due to the machining precision is, in the worst case, of the order of $0.15 \%$ of the applied displacement amplitude. The final distance between the two holders is checked after the shaft is removed. Note the high clearance and the spherical washers used between the upper holder of bellows and the lower plate of the setup (Fig. 5) that allow for any angular and radial misalignment. As mentioned in Section 2, the tests are displacement-controlled with a zero mean value, $\bar{u}=0$, and an amplitude $u_{a}$ of the bellows elongation, i.e., $\left(u_{m}-u_{f}\right)$.

A last check on bellows is performed by using an infrared CCD camera (CEDIP JADE MWIR with a resolution of $\pm 10 \mathrm{mK}$ ) to measure the temperature fields at one bellows surface covered with a black paint with an emissivity close to one [Arnould et al, 2005]. The camera acquisition frequency is slightly different from the loading frequency to obtain a stroboscopic effect. An example of the temperature fields is given in Figs. 10a and b at extreme elongations of the bellows. The temperature change arising from the thermoelastic coupling is clearly observed, i.e., the temperature variation is proportional to the variation in the stress-tensor trace. Figure 10b corresponds to a tension of the bellows, i.e., $\left(u_{m}-u_{f}\right)=0.48 \mathrm{~mm}$. In this case, the external fillet of 
the outside surface of a bellows wave is in compression whereas the internal ones are in tension (the reverse loadings occur at the inner surface of the bellows wave). An increase in the temperature of the external fillet and a decrease in the temperature of the internal fillet are then observed. The opposite observation is made when the bellows is in compression (Fig. 10a). The axisymmetry of the temperature fields in both cases and the temperature gradient through the bellows fillets show that each of them is equally loaded in pure tension. Let us note that a slight heating (less than $1^{\circ} \mathrm{C}$ in that case) of the bellows lower holder is observed in Figures 10a and b. This is due to micro-friction between disks and spacers in the (inner) clamp (see Section 4). This level is too small to have an effect on the fatigue properties of bellows.

The same bellows and infrared camera is used to assess the endurance limit by carrying out temperature measurements for different displacement amplitude steps with a maximum value of $0.48 \mathrm{~mm}$ [Arnould et al., 2005]. The endurance is estimated to be close to $0.265 \pm 0.065 \mathrm{~mm}$. Fatigue tests on eight bellows at the same time were then carried out (Fig. 4a) for a slightly greater displacement range, i.e., $0.35 \mathrm{~mm}$. Table 1 summarizes the results obtained. The load cell is mounted on bellows no. 9. An overpressure of about $800 \mathrm{kPa}$, which has a negligible effect on fatigue crack initiation, is applied to the bellows. Upon cycling, fluctuations less than $50 \mathrm{kPa}$ were observed with all the analyzed bellows. Airtightness loss in one bellows is detected when a pressure drop is observed, i.e., the experiment is automatically stopped when a pressure threshold (average value: $650 \mathrm{kPa}$ ) is reached. The number of additional cycles from airtightness loss to experiment stop is estimated if the crack propagation law [Arnould et al., 2004] and the air flow through it are known. However, this number of cycles is equal to less than $0.4 \%$ of the total number of cycles $N$ that is negligible when compared with the scatter of the results. In situ bellows load measurements did not reveal any drop, i.e., stiffness decrease, prior to initiation. Consequently, the pressure measurement was the key for monitoring airtightness. Last, when a broken bellows is detected, its mounting shaft (Fig. 6) is put back before the bellows is removed from the setup and the experiment restarts for the remaining samples after the overpressure is reapplied.

The number of cycles to airtightness loss varies from about 1 million to less than 6 million cycles for the eight tested bellows. As bellows in Table 1 are numbered in the same order as placed in the setup, it shows that the failures occurred at random locations. It demonstrates that the variation in cycles to failure is due to the stochastic nature of the bellows fatigue behavior and not to an experimental artifact. However, the lowest value, at $0.35 \mathrm{~mm}$ of displacement amplitude, is obtained for the bellows mounted on the piezoelectric load cell. This may be due to some mounting difficulties in this case that induce initial overload or damage to the bellows. Scanning electron microscopy (SEM) micrograph of bellows after airtightness loss shows that fatigue crack 
always occurs at the base of one inner fillet of the bellows (Fig. 10b), i.e., where the maximum principal stress level is the highest. This is another proof of the pure tensile loading of bellows. Last, at the end of these experiments, the setup properties (e.g., first natural frequency and stiffness) are measured one more time after more than $10^{7}$ cycles. No changes are observed. This validates the ability of the setup to perform high cycle fatigue test.

\section{Summary}

A specific fatigue test setup was designed when using a resonant fatigue machine. The designed setup was validated by performing static and dynamic tests. Pure axial displacement amplitudes of $0.5 \mathrm{~mm}$ with an uncertainty of about $10 \mu \mathrm{m}$ are achieved for a resonance frequency of $93 \mathrm{~Hz}$. It allows for an accurate and simultaneous test of eleven samples (at most) with a very low stiffness (e.g., about $2.65 \mathrm{~N} / \mathrm{mm}$ for $50 \mu \mathrm{m}$ thick bellows) in high cycle fatigue. In the present case, failure is detected during the experiment on bellows by airtightness loss through a pressure drop detection. The experimental data obtained on bellows will allow for the identification of a probabilistic model to assess their high cycle fatigue properties [Arnould et al., 2004; 2005].

\section{Acknowledgements}

The authors wish to thank Mr. Launay (SERAM Bordeaux, France) and Mr. Arnoux (Atmostat, France) for their invaluable help for the design of the setup and the Vibrophore reconditioning, and Mr. Fayolle for the implementation of the correction procedure.

\section{References}

Aktaa J, Reszat JTh, Walter M, Bade K, Hemker KJ (2005) High cycle fatigue and fracture behaviour of LIGA nickel. Scripta Materialia 52: 1217-1221.

Allameh SM, Lou J, Kavishe F, Buccheit T, Soboyejo WO (2004) An investigation of fatigue in LIGA Ni MEMS thin films. Material Science and Engineering A 371: 256-266.

Arnould O, Hild F (2002) Long-term life of Ni/Cu bellows: effect of diffusion on thermomechanical properties. Defect and Diffusion Forum 203-205: 61-80.

Arnould O, Hubert O, Hild F (2004) Thermomechanical properties and fatigue of nanocrystalline $\mathrm{Ni} / \mathrm{Cu}$ electrodeposits. MRS Symposium Proceedings 821: 357-362.

Arnould O, Brémond P, Hild F (2005) Fatigue limit evaluations of complex structure by using temperature measurements. Applied Mechanics and Materials 3-4: 71-76. 
Ashby MF (2005) Materials selection in mechanical design ( $3^{\text {rd }}$ ed.), Elsevier Science and Technology, Oxford, UK.

Barenblatt GI (1987) Dimensional analysis. Gordon \& Breach Science, New York, USA.

Boyce BL, Michael JR, Kotula PG (2004) Fatigue of metallic microdevices and the role of fatigue-induced surface oxides. Acta Materialia 54: 1609-1619.

Cho HS, Hemker KJ, Lian K, Goettert J, Dirras G (2003) Measured mechanical properties of LIGA Ni structures. Sensors and Actuators A 103: 59-63.

Giannakopoulos AE, Lindley TC, Suresh S (1998) Aspects of equivalence between contact mechanics and fracture mechanics: theoretical connections and life-prediction methodology for fretting-fatigue. Acta Materialia 46: 2955-2968.

Lou J, Shrotriya P, Soboyejo SW (2005) A cyclic microbend study on LIGA Ni microelectromechanical systems thin films. AMSE Journal of Engineering Materials and Technologies 127: 16-22.

Madou M (2002) Fundamentals of microfabrication: the science of miniaturization (2 ${ }^{\text {nd }}$ ed.), CRC Press, Boca Raton, Florida, USA.

Meirovitch L (2001) Fundamentals of vibrations, McGraw-Hill, New York, USA.

Mohr J, Strohrmann M (1992) Examination of long-term stability of metallic LIGA microstructures by electromagnetic activation. Journal of Micromechanics and Microengineering 2: 193-195.

Lord Rayleigh (1916) The theory of the Helmholtz resonator. Proceedings of the Royal Society of London A 92(638): 265-275.

Timoshenko SP, Woinowsky-Krieger S (1959) Theory of plates and shells (2 ${ }^{\text {nd }}$ ed.), McGraw-Hill, New York, USA.

Zwick-Roell (November, 2006) http://www.zwick.com/upload/machines_and_devices_EN. 


\section{Table}

Table 1: Experimental results on bellows. Number $N$ of loading cycles to airtightness loss for different displacement amplitude $u_{a}$. Bellows no. 1 has been used in a first experiment for thermal measurements (Figs. 10a and b). Bellows no. 2 to 9 have been tested simultaneously (Fig. 4a). Bellows no. 9 has been mounted with the piezoelectric load cell (Fig. 5b).

\begin{tabular}{|c|c|c|c|c|c|c|c|c|c|}
\hline Bellows no. & 1 & 2 & 3 & 4 & 5 & 6 & 7 & 8 & 9 \\
\hline$u_{a}(\mathrm{~mm})$ & 0.48 & 0.35 & 0.35 & 0.35 & 0.35 & 0.35 & 0.35 & 0.35 & 0.35 \\
\hline$N$ (10 cycles $)$ & 0.02 & 5.73 & 3.05 & 4.79 & 2.32 & 3.56 & 4.98 & 4.70 & 1.56 \\
\hline
\end{tabular}




\section{List of figures}

Figure 1: a) Schematic cross section view of a bellows with its stainless steel holders welded by a pulsed laser. b) Micrograph of $\mathrm{Ni} / \mathrm{Cu} / \mathrm{Ni}$ bellows cross-section obtained in an SEM after chemical etching.

Figure 2: a) Main view of the used resonant electromagnetic machine (Vibrophore) in its classical configuration (i.e., with a single cylindrical specimen). b) Operating principle. The resonance frequency of the sample (spring $\left.k_{s}\right)$-variable mass $\left(m_{v}\right)$ is driven by a magnet that prescribes the force amplitude $F_{a}$. The mean force $\bar{F}$ is obtained and controlled by the compression of a flexible joint. c) Schematic diagram of the setup to test $\ell$ bellows in parallel.

Figure 3: General schematic view of the high cycle fatigue setup. a) Half front cross-section. b) Top cut with one bellows (sample) mounted without any pneumatic fitting. The different screws or nuts used for the clamps at the end of the columns are not drawn for the sake of clarity.

Figure 4: a) View of the setup mounted in the Vibrophore during experiments on bellows. b) View of the free core LVDT (as shown by the arrow in Fig. 4a).

Figure 5: Installation sketch of the bellows on the upper and lower plates of the setup (Fig. 3b). a) Standard configuration with a pneumatic fitting to measure airtightness loss. b) Specific configuration with a piezoelectric load cell to measure the bellows stiffness.

Figure 6: Detailed view of the bellows mounting setup. a) Sample with its bellows welded onto holders. A specific shaft is used during welding and mounting in the fatigue setup to ensure an initial stressfree state of the bellows. b) Corresponding cross-section.

Figure 7: Static validation of the setup. a) View of the setup in a servohydraulic testing machine. Two quasi-static LVDTs measure the relative axial displacements between the lower and upper plates of the setup. b) Setup load vs. relative displacement of the LVDTs.

Figure 8: Dynamic validation of the setup. a) Global view with mock-up samples that simulate the bellows behavior. b) Upper and lower markers (as shown by the box in Fig. 8a) whose relative displacements are visualized with a strobe light and a long distance microscope. c) View (as shown by the arrow in Fig. 8a) of the load cell assembly on the upper holder of a spring and the accelerometer to correct dynamic effects (Fig. 9). 
Figure 9: $\quad$ Load measurement correction. a) Frequency spectrum of the load measured by the piezoelectric cell on mock-up samples (Figs. 8a and c). b) Raw force vs. displacement curve for a mock-up sample and corrected response.

Figure 10: Temperature fields $\left({ }^{\circ} \mathrm{C}\right)$ on the bellows surface measured by an infrared camera for $u_{a}=0.48 \mathrm{~mm}$ and $f=93 \mathrm{~Hz}$. The picture corresponds to the box defined in Fig. $6 \mathrm{a}$ for the maximum contraction (a) and extension (b) of the bellows. c) SEM micrograph of a fatigue crack in bellows for $u_{a}=0.35 \mathrm{~mm}$. 


\begin{tabular}{|c|c|c|c|c|c|c|c|c|c|}
\hline Bellows no. & 1 & 2 & 3 & 4 & 5 & 6 & 7 & 8 & 9 \\
\hline$u_{a}(\mathrm{~mm})$ & 0.48 & 0.35 & 0.35 & 0.35 & 0.35 & 0.35 & 0.35 & 0.35 & 0.35 \\
\hline$N\left(10^{6}\right.$ cycles $)$ & 0.02 & 5.73 & 3.05 & 4.79 & 2.32 & 3.56 & 4.98 & 4.70 & 1.56 \\
\hline
\end{tabular}

Table 1: Experimental results on bellows. Number $\boldsymbol{N}$ of loading cycles to airtightness loss for different displacement amplitude $u_{a}$. Bellows no. 1 has been used in a first experiment for thermal measurements (Figs. 10a and b). Bellows no. 2 to 9 have been tested simultaneously (Fig. 4a). Bellows no. 9 has been mounted with the piezoelectric load cell (Fig. 5b).

$125 \times 21 \mathrm{~mm}(600 \times 600 \mathrm{DPI})$ 


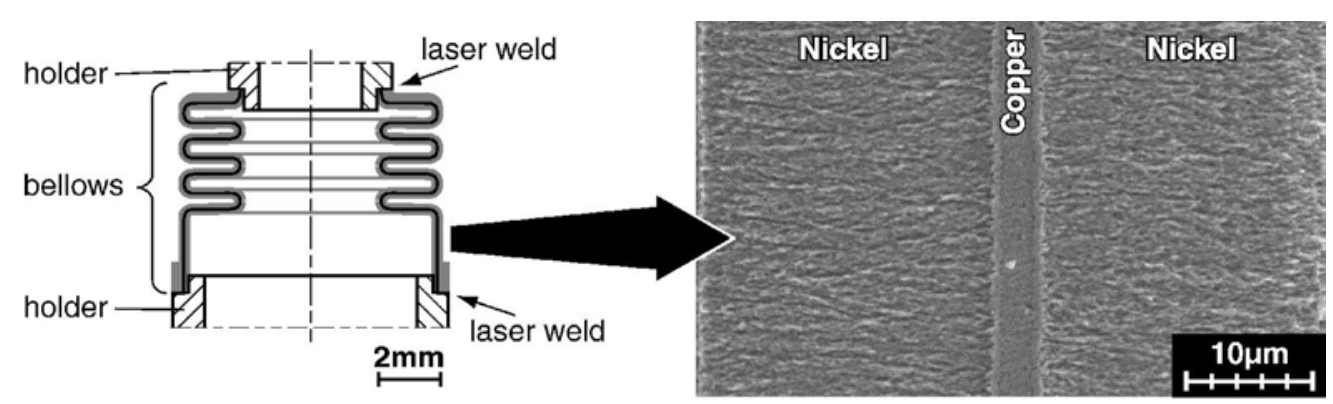

(a)

(b)

Figure 1: a) Schematic cross section view of a bellows with its stainless steel holders welded by a pulsed laser. b) Micrograph of $\mathrm{Ni} / \mathrm{Cu} / \mathrm{Ni}$ bellows cross-section obtained in an SEM after chemical etching. $151 \times 49 \mathrm{~mm}(600 \times 600 \mathrm{DPI})$ 


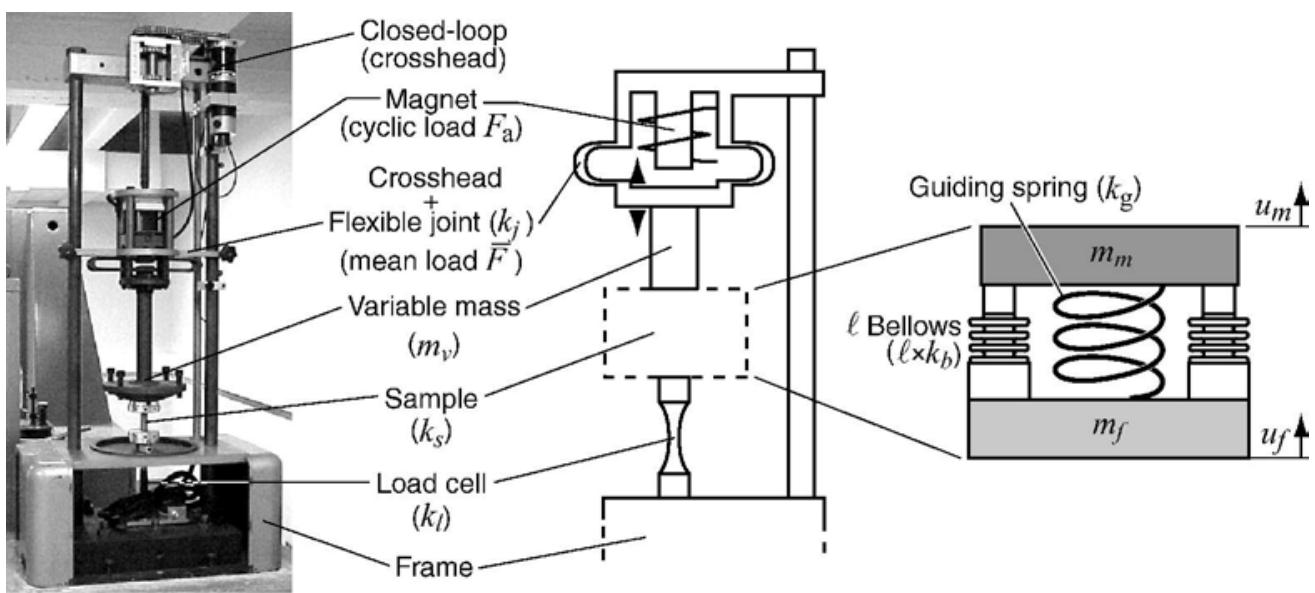

(a)

(b)

(c)

Figure 2: a) Main view of the used resonant electromagnetic machine (Vibrophore) in its classical configuration (i.e., with a single cylindrical specimen). b) Operating principle. The resonance frequency of the sample (spring $k_{s}$ ) - variable mass $\left(m_{v}\right)$ is driven by a magnet that prescribes the force amplitude $F_{a}$. The mean force $F$ is obtained and controlled by the compression of a flexible joint. c) Schematic diagram of the setup to test $I$ bellows in parallel.

$165 \times 79 \mathrm{~mm}(600 \times 600 \mathrm{DPI})$ 


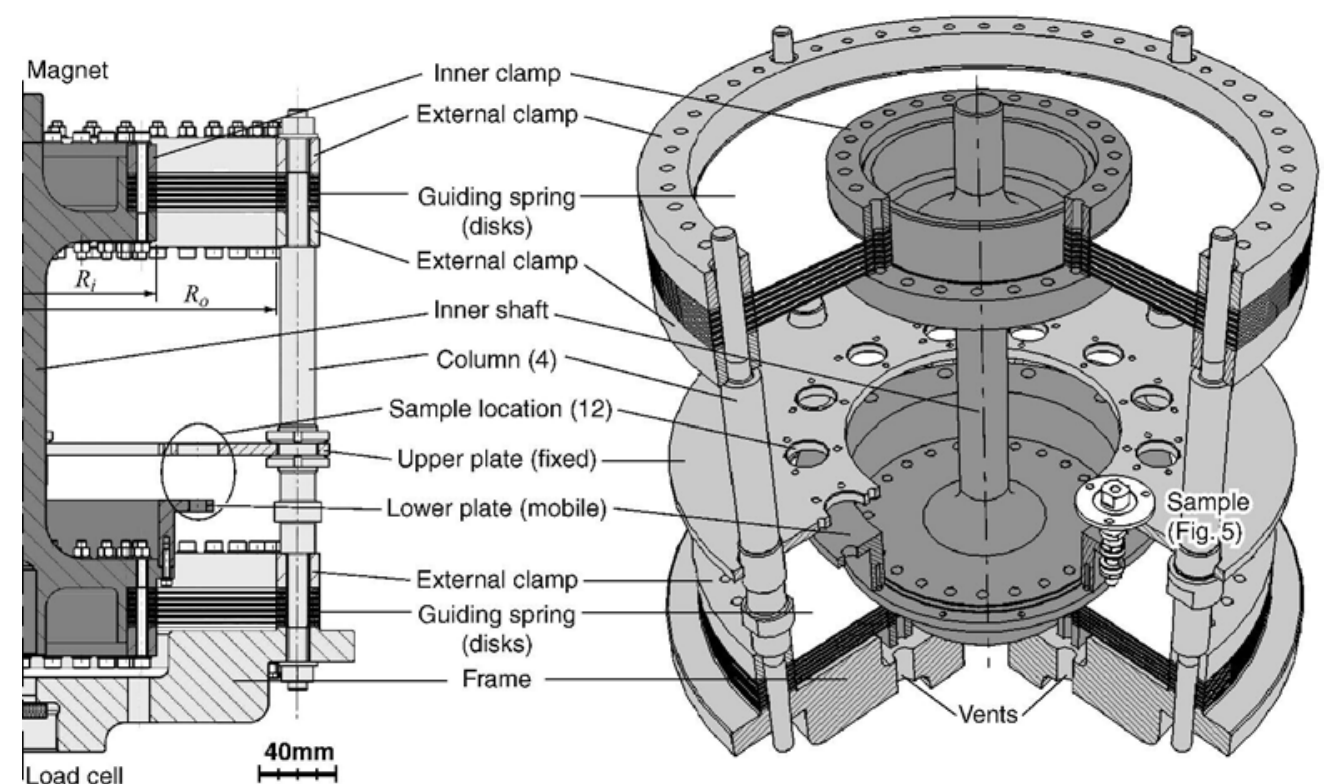

(a)

(b)

Figure 3: General schematic view of the high cycle fatigue setup. a) Half front crosssection. b) Top cut with one bellows (sample) mounted without any pneumatic fitting. The different screws or nuts used for the clamps or at the end of the columns are not drawn for the sake of clarity. $187 \times 116 \mathrm{~mm}(600 \times 600 \mathrm{DPI})$ 


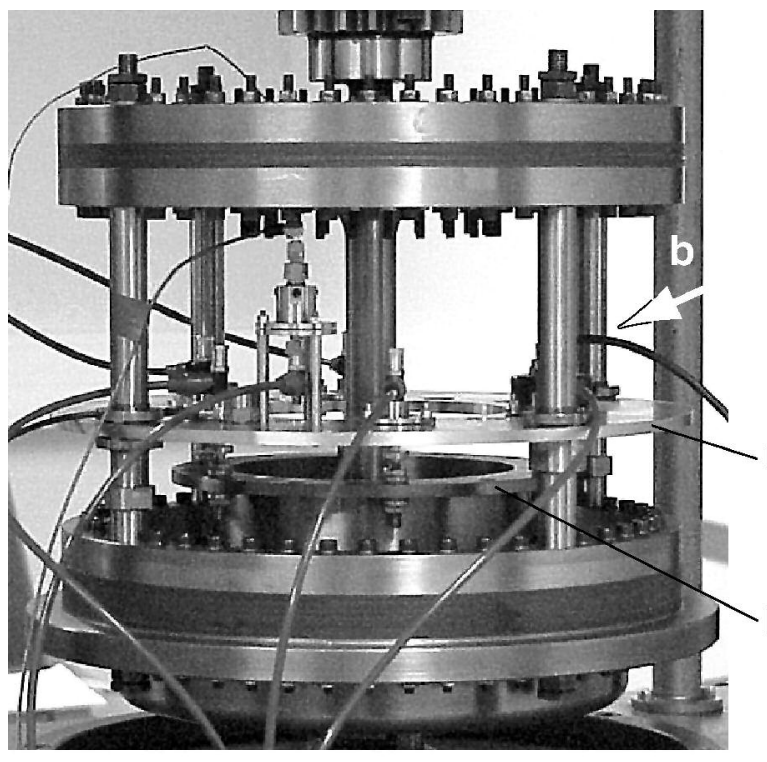

(a)

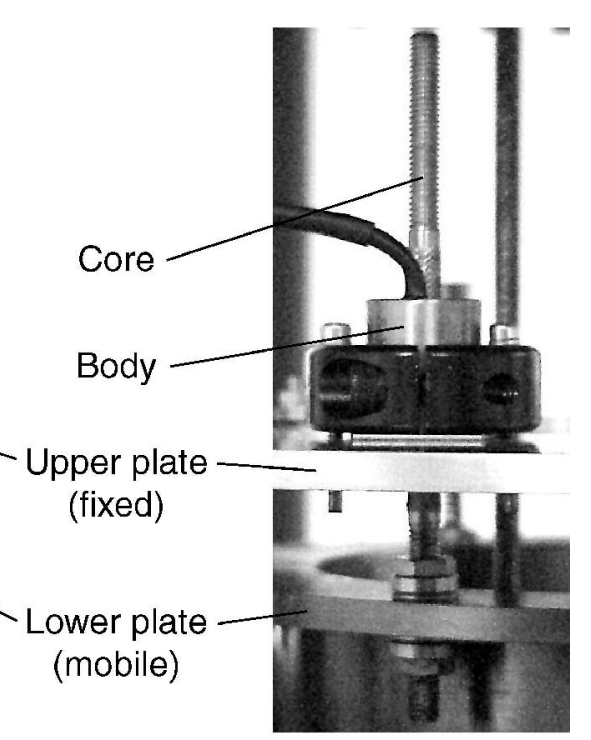

(b)

Figure 4: a) View of the setup mounted in the Vibrophore during experiments on bellows. b) View of the free core LVDT (as shown by the arrow in Fig. 4a). $129 \times 79 \mathrm{~mm}(600 \times 600 \mathrm{DPI})$ 


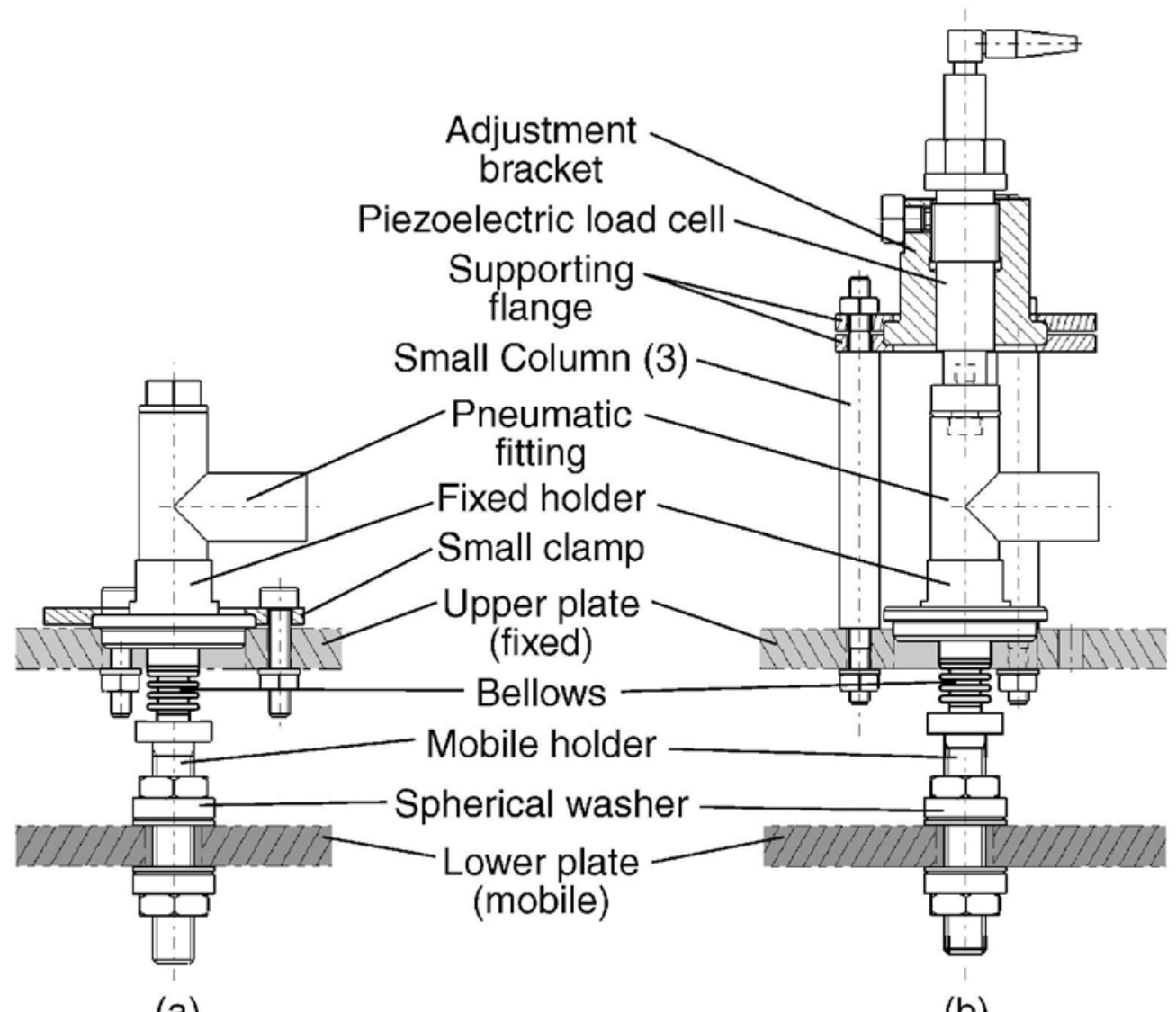

(a)

(b)

Figure 5: Installation sketch of the bellows on the upper and lower plates of the setup (Fig. 3b). a) Standard configuration with a pneumatic fitting to measure airtightness loss.

b) Specific configuration with a piezoelectric load cell to measure the bellows stiffness. $107 \times 94 \mathrm{~mm}(600 \times 600 \mathrm{DPI})$ 
(a)

(b)

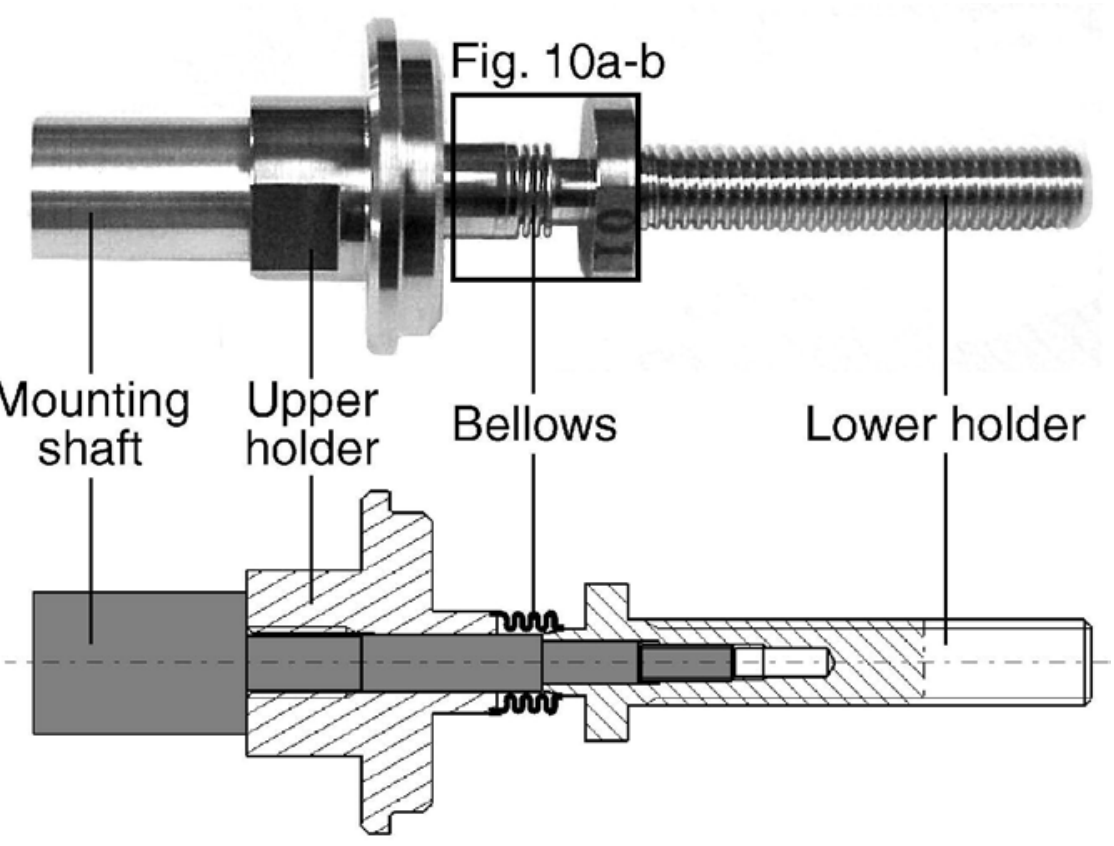

Figure 6: Detailed view of the bellows mounting setup. a) Sample with its bellows welded onto holders. A specific shaft is used during welding and mounting in the fatigue setup to ensure an initial stress-free state of the bellows. b) Corresponding cross-section. $94 \times 60 \mathrm{~mm}(600 \times 600 \mathrm{DPI})$ 


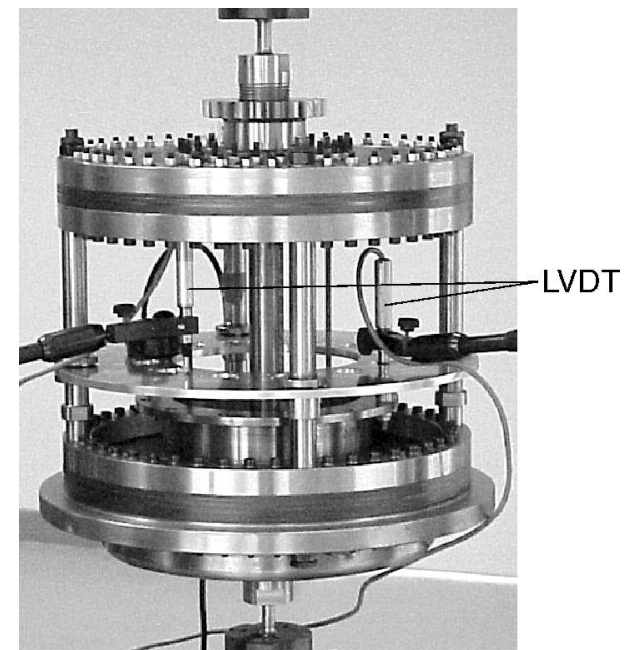

(a)

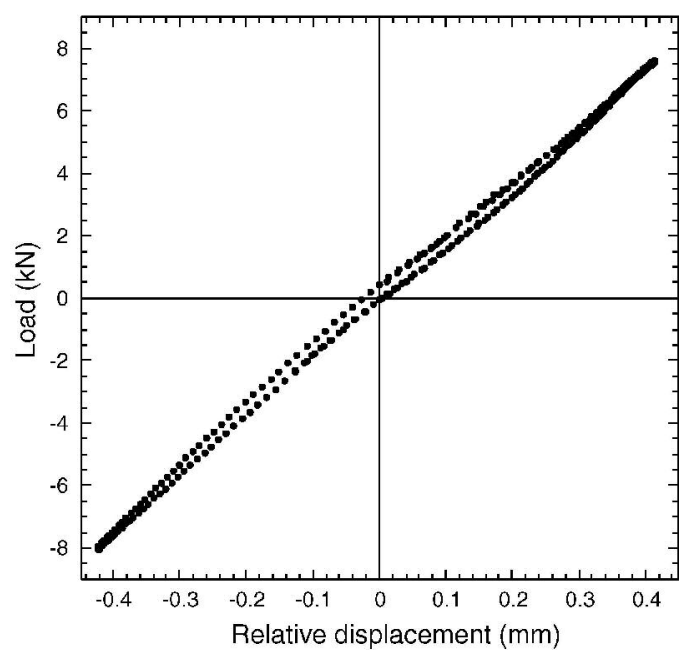

(b)

Figure 7: Static validation of the setup. a) View of the setup in a servohydraulic testing machine. Two quasi-static LVDTs measure the relative axial displacements between the lower and upper plates of the setup. b) Setup load vs. relative displacement of the LVDTs. $172 \times 91 \mathrm{~mm}(600 \times 600 \mathrm{DPI})$ 


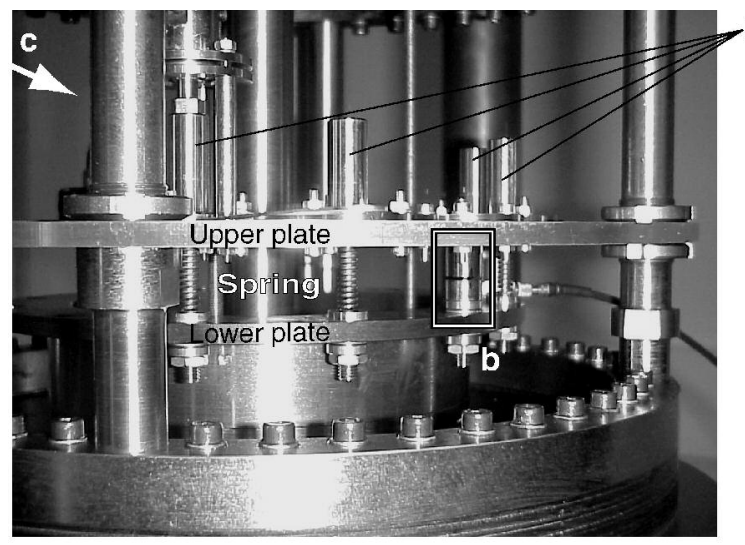

(a)

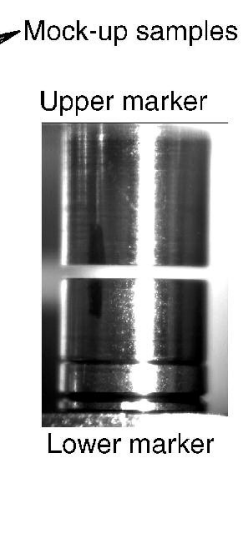

(b)

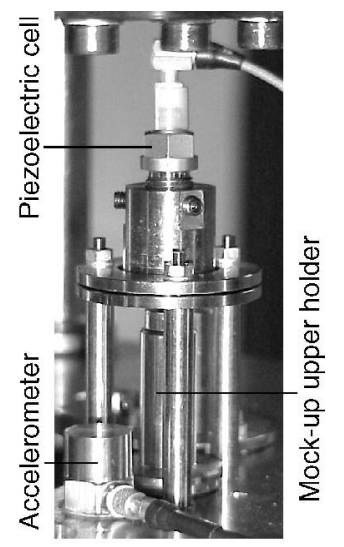

(c)

Figure 8: Dynamic validation of the setup. a) Global view with mock-up samples that simulate the bellows behavior. b) Upper and lower markers (as shown by the box in Fig. 8a) whose relative displacements are visualized with a strobe light and a long distance microscope. c) View (as shown by the arrow in Fig. 8a) of the load cell assembly on the upper holder of a spring and the accelerometer to correct dynamic effects (Fig. 9). $167 \times 74 \mathrm{~mm}(600 \times 600 \mathrm{DPI})$ 


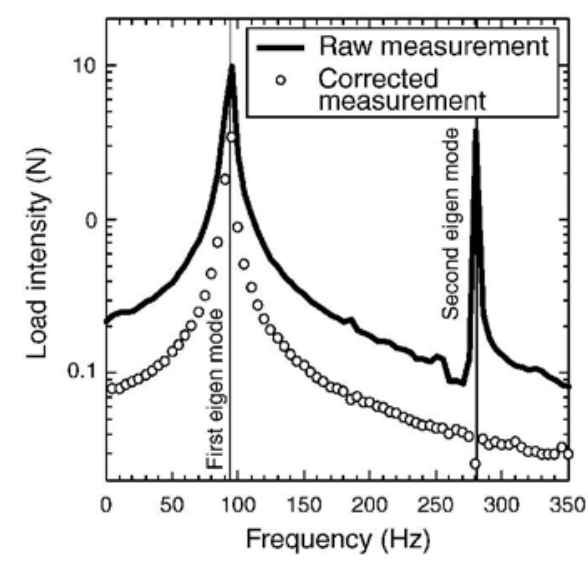

(a)

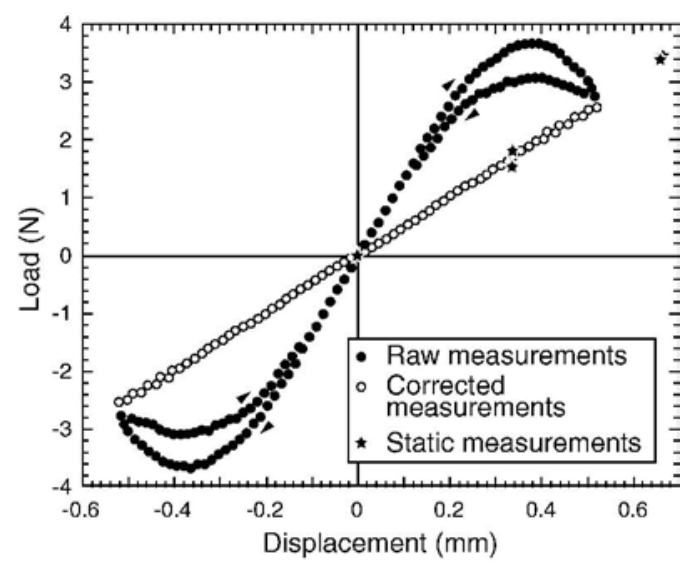

(b)

Figure 9: Load measurement correction. a) Frequency spectrum of the load measured by the piezoelectric cell on mock-up samples (Figs. 8a and c). b) Raw force vs. displacement curve for a mock-up sample and corrected response.

$168 \times 76 \mathrm{~mm}(600 \times 600 \mathrm{DPI})$ 


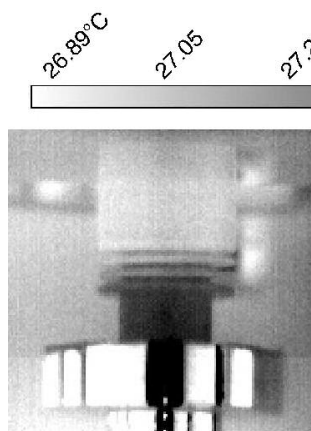

(a)
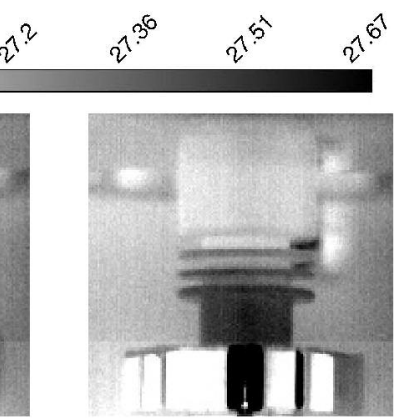

(b)

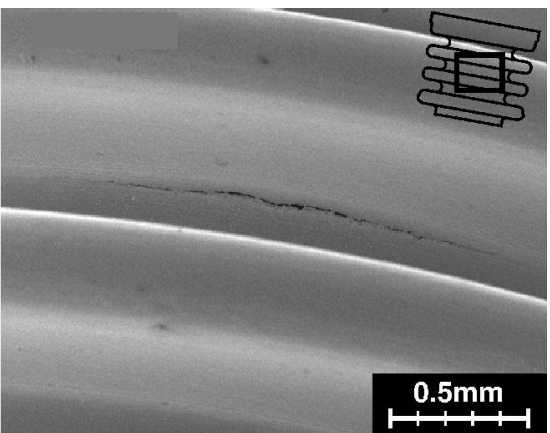

(c)

Figure 10: Temperature fields $\left({ }^{\circ} \mathrm{C}\right)$ on the bellows surface measured by an infrared camera for $u_{a}=0.48 \mathrm{~mm}$ and $f=93 \mathrm{~Hz}$. The picture corresponds to the box defined in Fig. 6a for the maximum contraction (a) and extension (b) of the bellows. c) SEM micrograph of a fatigue crack in bellows for $u_{a}=0.35 \mathrm{~mm}$.

$160 \times 59 \mathrm{~mm}(600 \times 600 \mathrm{DPI})$ 